\section{OP13 UNDERSTANDING SOCIAL INEQUALITIES IN CHILDHOOD UNINTENTIONAL INJURIES: FINDINGS FROM THE UK MILLENNIUM COHORT STUDY}

${ }^{1} \mathrm{M}$ Campbell ${ }^{*},{ }^{2} \mathrm{~A}$ Pearce, ${ }^{3} \mathrm{E}$ Orton, ${ }^{1} \mathrm{~S}$ Wickham, ${ }^{3} \mathrm{D}$ Kendrick, ${ }^{1} \mathrm{D}$ Taylor-Robinson. ${ }^{1}$ Department of Public Health and Policy, Liverpool University, Liverpool, UK; ' Institute of Child Health, University College London, London, UK; ${ }^{3}$ Division of Primary Care, University of Nottingham, Nottingham, UK

10.1136/jech-2018-SSMabstracts. 13

Background Childhood unintentional injuries (UI) are common with stark health inequalities. Understanding the pathways to inequalities in childhood UIs is essential in tackling this public health challenge. Using a national birth cohort, the aim of this study was to assess the role of early-life risk factors in explaining excess UIs in children from lower income households.

Methods Analysis of the United Kingdom (UK) Millennium Cohort Study ( 18000 children born 2000-2002), based on 14335 who participated in three sweeps, at ages 9 months, 3 and 5 years. Our main exposure measure of socio-economic circumstances (SEC) was household income quintile at birth. Our binary outcome was derived from parent-reported ever had an accident and was taken to the doctor, health centre or hospital at 5 years old since the last sweep. Relative risk (RRs) and 95\% confidence intervals (95\% CI) for UIs were estimated using Poisson regression, by household income quintile. Potential early-life risk factors were explored to assess if they attenuated the associations between family income and UI in models adjusting for factors within three domains: environmental safety, supervision and child behaviours and abilities. Analyses were conducted using Stata/SE with svy commands to account for the sampling design and attrition using survey weights. We repeated the analysis using an alternative outcome (UI admissions), SEC exposure (maternal education) and using multiple imputations to address missingness.

Results $28 \%$ of children had a UI from 3 to 5 years old. Children in the lowest income quintile had a $20 \%$ greater risk of UIs compared to those in the highest (aRR 1.20 95\% CI $1.05,1.37)$. Sequentially adjusting for factors that may influence: environmental safety (aRR 1.18, 95\% CI 1.01,1.37), supervision (aRR 1.17, 95\% CI 1.00,1.36) and child behaviours and abilities (aRR 1.14 95\% CI 0.98,1.33) reduced the relative risk by $10 \%, 15 \%$ and $30 \%$ respectively, rendering the association between income and UI non-significant in our final model. Our sensitivity analyses support our main findings.

Conclusion Using a nationally representative sample, we found one in four UK children had a UI from 3 to 5 years old, with significant inequalities. These were partly explained by factors that may influence: environmental safety, supervision and child behaviour and abilities. Interventions to address these potentially modifiable risk factors may also reduce inequalities in childhood injury. Our use of 'proxy' measures is a limitation of this study. Future research needs to further understand how these factors may influence UI.

\section{OP14 HOW WELL CAN EARLY CHILD DEVELOPMENT BE PREDICTED IN CHILDREN IN THE UK? FINDINGS FROM THE MILLENNIUM COHORT STUDY}

CL Camacho*, VS Straatmann, JC Day, DC Taylor-Robinson. Public Health and Policy, University of Liverpool, Liverpool, UK

\subsection{6/jech-2018-SSMabstracts. 14}

Background Early cognitive development (ECD) is an important determinant of educational attainment and health outcomes. ECD is socially patterned and influenced by biological, environmental and socioeconomic factors. This study aimed to identify how well socio-economic and demographic factors from the first year of life predicted ECD at age 3 in a contemporary UK cohort.

Methods We used data on 9487 complete cases children from the UK Millennium Cohort Study collected at 9 months and 3 years old. The outcome was school readiness using the Bracken School Readiness Assessment (BSRA) assessed at age 3 years. Predictive risk modelling (PRM) was carried out and the discriminatory capacity of two models was compared by the area under the receiver operating characteristic curve (AUROC; 95\% Confidence Intervals). Stepwise statistical selection specified a model with 13 perinatal and sociodemographic predictors collected at age 9 months. This was compared with a parsimonious model comprising the top 6 predictors, identified by dominance analyses which ranks the importance of each variable in the model. Integrated discrimination improvement (IDI) was also calculated, comparing the two models. All analyses were conducted in Stata SE version 14.2.

Results At age 3, 11.7\% (11.0\%-12.3\%) of children were not school ready. The 13 variables statistically selected were: parents' National Statistics Socio-Economic Classification, child's ethnic group, maternal education, income band, sex, number of children in the household, mother's age, low birth weight, mother's mental health, infant developmental milestones, breastfeeding, parents' employment, housing type. The parsimonious model included the first six listed variables, based on dominance analyses. The AUROC for the full model (13 predictors) was $0.80(0.78-0.81)$ and $0.78(0.77-0.79)$ for the reduced model (6 predictors). IDI showed there was a small but significant difference in performance, with the full model resulting in a $1.3 \% \quad(\mathrm{p} \leq 0.001)$ improvement in discrimination.

Conclusion We identified a set of predictive risk factors from the perinatal period and early infancy that predicted school readiness at age 3 with good discrimination. Social factors were the strongest predictors of school readiness. This study demonstrates the feasibility of predicting school readiness using just six attributes collected around the time of birth. PRMs could be used to identify children who would potentially benefit most from early interventions. Further research to assess the impact of this PRM would be required before it could be used in practice. A strength is the nationally representative sample; a limitation is the lack of external validation. 